\title{
MEDIACIONES INDÍGENAS EN EL ESPACIO AUDIOVISUAL: PELÍCULAS, SERIES Y VIDEOCLIPS
}

\author{
INDIGENOUS MEDIATIONS IN THE AUDIOVISUAL GROUND: FILMS, SERIES \\ AND VIDEO CLIPS
}

Gabriel Izard Martínez ${ }^{1}$

Universidad de Barcelona

Juan Antonio Flores Martos ${ }^{2}$

Universidad de Castilla-La Mancha

Mònica Martínez Mauri ${ }^{3}$

Universitat de Barcelona. Serra Húnter Fellow

Recibido: 21 de marzo de 2021; Aprobado: 11 de octubre de 2021.

Cómo citar este artículo / Citation: Izard Martínez, Gabriel, Juan Antonio Flores Martos y Mònica Martínez Mauri. 2021. "Mediaciones indígenas en el espacio audiovisual: películas, series y videoclips". Disparidades. Revista de Antropología 76(2): e015d. doi: <https://doi.org/10.3989/dra.2021.015d>.

RESUMEN: En este artículo presentamos tres casos de jóvenes artistas indígenas de México y Panamá que, desde el cine, la televisión y el rap, y combinando su poética con la agencia política, crean contenidos novedosos para audiencias indígenas y no indígenas globales. Argumentamos que estos comunicadores de la era digital pueden ser considerados nuevos mediadores que, a través de un espacio intermedio audiovisual, visibilizan ante sus comunidades y el mundo una nueva realidad indígena.

PALABRAS CLAVE: Pueblos indígenas; Cine; Televisión; Música; Comunicación; Mediación.

\begin{abstract}
In this paper we present three cases of young indigenous artists from Mexico and Panama. Through film, television and rap, they combine their poetics with political agency, creating novel content for global indigenous and non-indigenous audiences. We argue they can be regarded as new mediators who make visible to their communities and the world a new indigenous reality from a middle audiovisual ground.
\end{abstract}

KEYWORDS: Indigenous Peoples; Cinema; Television; Music; Communication; Mediation.

1 Correo electrónico: gabrielizard@ub.edu. ORCID iD: <https://orcid.org/0000-0002-8254-1833>.

2 Correo electrónico: juanantonio.flores@uclm.es. ORCID iD: <https://orcid.org/0000-0003-1642-1937>.

3 Correo electrónico: martinezmauri@ub.edu. ORCID iD: <https://orcid.org/0000-0003-1857-1146>. 


\section{INTRODUCCIÓN}

Coleman (2010) se sirve del concepto de cronopolítica alocrónica (allochronic chronopolitics) para explicarnos que seguimos dominados por la idea de que los Otros viven en una dimensión temporal distinta y que la era digital sirve para construir nuevos muros. En este sentido pareciera que las sociedades indígenas se hubieran quedado al margen de este mundo dominado por los medios digitales y su vida transcurriera sin entrar a Facebook, WhatsApp o Instagram (López García 2012). Sin embargo, muchos indígenas no solo usan los celulares e Internet, sino que producen y difunden contenidos digitales a través de ellos ${ }^{4}$. Se trata de individuos que podrían ser definidos como mediadores o que ocuparían lo que el historiador White denominó middle ground (espacio intermedio): un mundo construido en el cruce de caminos, mutuamente comprensible, caracterizado por nuevos sistemas de significado e intercambio, en el que las relaciones van más allá de las ideas de dominación, subordinación y aculturación (White 1991: ix). Aunque White desarrolló este concepto para hablar de las relaciones entre los blancos y los indígenas en la región de los Grandes Lagos de Norteamérica durante los siglos XVII-XIX, esta idea parece pertinente para abordar las creaciones audiovisuales por parte de jóvenes indígenas en la Latinoamérica de hoy.

A finales del siglo XX Conklin y Graham (1995) evidenciaron el desarrollo de un nuevo middle ground entre algunos ciudadanos del cuarto $y$ del primer mundo. Un espacio intermedio que no se materializaba en un territorio geográfico, ni en un espacio social donde los protagonistas se encontraban cara a cara, sino que se trataba de un espacio político, un ámbito de comunicación intercultural, de intercambio y de acción política conjunta transnacional. Pero un espacio que dependía de la mediación de unos pocos individuos con competencias lingüísticas, logísticas y técnicas. En este artículo nos preguntamos hasta qué punto la emergencia y consolidación del espacio web 2.0 marca un antes y un después en estas mediaciones. ¿Qué elementos introducen los nuevos medios en las mediaciones? ¿Qué impacto tienen dentro

4 En cuanto a la creación de nuevos medios indígenas, cfr. Orobitg 2020a y 2020b. y fuera de las comunidades? Para intentar dar respuesta a estos interrogantes nos centramos en la experiencia de jóvenes emberás, dules ${ }^{5}$ y totonacos dedicados a la producción audiovisual.

La literatura antropológica sobre jóvenes artistas indígenas desde la perspectiva de su liderazgo como mediadores no es demasiado abundante (Evans 2012; Kenny y Fraser 2013; Evans y Sinclair 2016). Este artículo se centra además en un tipo de análisis que va más allá de sus roles políticos, poniendo el foco en la combinación de su arte (poética) y potencia política. Dicha combinatoria es probablemente la razón de que se hayan enfrentado de forma exitosa al reto de comunicar y mediar entre su «comunidad» (cualesquiera que ésta sea) y un mundo indígena y no indígena global.

\section{CREATIVIDAD Y REIVINDICACIÓN EN LA OBRA DE IVÁN JARIPIO Y EL FESTIVAL JUMARA}

Los cortometrajes de Iván Jaripio, joven cineasta emberá panameño, giran alrededor de la demanda de los derechos territoriales, los cuales constituyen uno de los ejes principales de la agenda política de los emberá y de los otros grupos indígenas de Panamá y de América Latina. En algunos casos Iván lo hace de manera más explícita, mostrando los estragos ecológico-culturales de la deforestación (la desaparición de los bosques, los ríos, los animales, así como las plantas medicinales y el fruto con cuyo tinte se realizan las pinturas corporales), como en «Arimae» $(2015)^{6}$, o tratando la reivindicación de la titulación colectiva de las tierras asignadas tras la reubicación provocada por la construcción de la represa en la Cuenca del Lago Bayano, como en "Dadji De» (Nuestro hogar, con Detsy Barrigón, $2014)^{7}$. Y en otros a través de metáforas en las que la desaparición de las pinturas corporales de un cuerpo femenino a consecuencia de la lluvia nos habla de la pérdida de la cultura provocada por la destrucción de la naturaleza, como en "Identidad» $(2016)^{8}$. De una forma o de otra, en sus películas, que han participado en diversos festivales nacionales e internacionales y obtenido premios, el

5 En este texto «dule», "guna» y "gunadule» son utilizados como sinónimos.

6 <https://vimeo.com/123862156>.

7 <http://www.wapikoni.ca/films/nuestro-hogar>.

8 <https://vimeo.com/269560314>. 
tema principal es siempre el binomio inseparable territorio-cultura.

La obra de Iván Jaripio tiene una doble dimensión creativa y reivindicativa, representativa de la doble faceta poética (Salazar y Córdova 2008) y política (Ginsburg 2002) del cine indígena. Esta dualidad también se observa en cada edición de Jumara («todos» en emberá), el festival internacional de cine indígena que Iván organiza en su comunidad de Piriatí Emberá, en la Cuenca del Bayano, desde 2018, cuando sintió la necesidad de llevar este cine, normalmente exhibido en capitales y grandes ciudades, a una comunidad indígena. En el evento se exhiben películas que tratan la salvaguarda del territorio ante la amenaza del Estado y las empresas capitalistas, la necesidad de preservación de la cultura y la defensa de los derechos humanos ante las injusticias y la violencia (los tres ejes narrativos principales del cine indígena); y por otra parte se desarrolla, sobre todo en las sesiones de inauguración y clausura, una verdadera performance de la identidad emberá: espectáculos de música y danza seguidos con emoción, de manera participativa, por los habitantes del pueblo, que acuden con las vestimentas tradicionales y las pinturas corporales referidas anteriormente (Gómez Ruiz e Izard Martínez 2020).

Sus películas y el festival que organiza hacen de Iván un comunicador, cuya labor se ve acrecentada por la difusión de su obra y de Jumara en las redes sociales: su Facebook personal y el del festival contienen links a su obra en Vimeo, así como otros materiales visuales y noticias del mundo indígena.

Cabe resaltar su importancia como mediador que, desde la creación y la reivindicación, da a conocer al mundo exterior la cultura emberá y sus demandas. Esta posición le confiere una especial visibilidad no solo hacia afuera, sino también entre su gente. Como muestra Terence Turner (1992: 6-7) para el caso kayapó en Brasil, la realización de videos o películas adquiere una especial significación social y política a partir del prestigio que tal actividad confiere y del hecho de que constituya una importante forma de mediación con el mundo no indígena. En relación con esto, Iván sería un representante de los nuevos roles derivados de la comunicación indígena, de una nueva generación de activistas comunicadores que, en este caso desde la producción de cortometrajes y la celebración de festivales, ejercen de portavoces de la realidad, las problemáticas y las reivindicaciones de sus grupos. Habría que añadir en este grupo a otros jóvenes emberá que ponen en contacto su cultura con el exterior desde ámbitos distintos como son las empresas turísticas comunitarias y los eventos intercomunitarios como los Juegos Ancestrales Emberá clasificatorios para los Juegos Mundiales de los Pueblos Indígenas. La conexión con el afuera no solo se da en estos casos a partir de esas iniciativas en sí mismas, sino también a través de formatos virtuales de difusión como páginas web y perfiles de facebook.

Estos mediadores digitales son el complemento, en los nuevos tiempos tecnológicos, de unos liderazgos que, en el caso emberá, han estado siempre relacionados con la reivindicación de derechos territoriales, iniciada en el Darién en la década de 1960 en el contexto de la competencia por la tierra con campesinos colonos, e intensificada con el apoyo del régimen de Omar Torrijos (Herlihy 1986; Colin 2010). De hecho Iván es hijo de un destacado líder de Piriatí implicado en la demanda de titulación colectiva de las tierras asignadas tras la construcción de la represa del Bayano. La poética cinematográfica y su difusión a través de los festivales y las redes sociales, van de la mano con la política de las organizaciones que luchan por el manejo autónomo y comunitario del territorio.

\section{CINE DOCUMENTAL, PELÍCULAS Y SERIES: PROYECTANDO GUNAYALA AL MUNDO}

En Gunayala, una de las autonomías indígenas más consolidadas del planeta, siempre han existido mediadores. Tradicionalmente estas personas eran denominadas siggwi (pájaro) y ocupaban un lugar destacado en las comunidades. Se trataba de personas que favorecían transferencias y diálogos entre universos aparentemente distintos, que atraían recursos hacia el ámbito local situándose en el centro de una extensa red social y que podían promover redefiniciones identitarias. Mientras que a principios del siglo XX los siggwi solían ser los secretarios de las saglas (jefes), a partir de los años 1980 se transformaron en asesores, directores de ONG o profesionales indígenas al servicio de las autoridades locales o comarcales (Martínez Mauri 2011).

Actualmente, en un contexto cada vez más marcado por la emergencia del espacio Web 2.0. y los contenidos audiovisuales, los hermanos Orgun y Duiren Wagua, profesionales dules dedicados 
a la creación audiovisual, median las relaciones entre Gunayala y el mundo. Ambos cuentan con formación en el exterior -Orgun en la Escuela Internacional de Cine y Televisión de Cuba (20082011), Duiren en Guatemala-, han colaborado con grandes producciones internacionales y trabajan con las comunidades indígenas construyendo relatos audiovisuales. En 2006 Orgun se integró al grupo Igar Yala. Junto a otros jóvenes colaboró con la estadounidense Veronica Bollow en el guión y filmación de la multigalardonada película Burwa Dii Ebo (El viento y el agua) (2008) ${ }^{9}$. Centrada en la vida de Machi, dule criado en la comarca y Rosy, en la urbe, el film narra la historia de su encuentro en un contexto marcado por la construcción de la carretera que conectará Gunayala con la ciudad y el desarrollo del turismo. Burwa Dii Ebo retrata un mundo en transformación, cada vez más expuesto a la interacción con el mundo urbano y a las inversiones extranjeras.

Orgun cuenta con una trayectoria profesional a nivel nacional que lo ha llevado a trabajar en el Servicio Estatal de Radio y Televisión (SERTV), colaborar con cineastas panameños y producir un documental sobre una de las figuras más olvidadas de la historia panameña: Victoriano Lorenzo. En El Héroe Transparente ${ }^{10}$, a través del testimonio de destacados activistas sociales y de la cultura, se reconstruye la memoria histórica sobre este campesino rebelde de origen buglé.

Duiren se formó como fotógrafo, estudió periodismo y en 2014 fue seleccionado en el 2. Festival Internacional de Cine Documental Acampadoc para desarrollar su primer cortometraje. Desde entonces ha trabajado en varios proyectos y está concluyendo Bila Burba (Alma revolucionaria), largometraje sobre la Revolución Dule, editado por su hermano. Duiren es el enlace de la CLACPI (Coordinadora Latinoamericana de Cine y Comunicación de los Pueblos Indígenas) para Panamá y en 2018 fue

9 Fue la primera película panameña proyectada en el prestigioso Festival de Cine Sundance (Estados Unidos) y ganó el Premio a Mejor Película Dramática de Largometraje y el Premio Mejor Película en Lenguaje Originario en el Imagine Native Festival de Toronto, Canadá, el premio del Jurado y el premio Signis a la Mejor Película Latinoamericana en el Festival SanFic 5, de Santiago de Chile.

10 <https://vimeo.com/126574485>. invitado por los productores a participar en el rodaje en Gunayala de la tercera temporada de La Casa de Papel, una de las series más exitosas de Netflix. Después de obtener el permiso del Congreso General Guna y pagar la suma que las autoridades comarcales y locales determinaron, en diciembre de 2018 se inició el rodaje en el sector occidental de la comarca. Duiren, además de leer el guión, sugerir algunos cambios, introducir alguna frase en lengua guna, se aseguró, junto a otros participantes dules, de que se respetaran los acuerdo firmados con el Congreso y que el nombre Gunayala apareciera en todas las secuencias grabadas en las islas.

Paradójicamente, la serie -proyectada en más de 100 países y con audiencias millonarias- no solo mostró que los gunas habían logrado construir una nación dentro de un Estado y habían mantenido su territorio insular fuera del alcance de grandes inversionistas extranjeros, sino que se convirtió en el mayor reclamo publicitario para sus prístinas y exóticas islas. Pronto llegaron miles de turistas ansiosos por pisar las localizaciones de la serie y tomarse una foto bajo una palmera con la máscara de Dalí.

Después de la experiencia de La Casa de Papel, Orgun, Duiren y otro socio guna, Machi Díaz, crearon una productora para desarrollar proyectos de cine y televisión tanto en la capital como en territorios indígenas. Con ella se proponen formar a una nueva generación de documentalistas. Además, gracias a su experiencia asesoran a otros equipos de filmación para la obtención de permisos, favoreciendo así la creación de un middle ground en torno a la producción de contenidos audiovisuales.

A través de sus producciones, los jóvenes de Wagua Films proyectan una realidad, la de sus comunidades de origen y la de otros pueblos, que tiene efectos sobre el día a día de miles de personas. Su mediación, no solo es sobre la representación de Gunayala hacia fuera, sino que cada vez tienen más influencia sobre la producción audiovisual en el territorio guna. Su manera de hacer cine, colaborando con los comuneros, dando voz a los protagonistas de la historia, negociando lo que se puede y lo que no se puede filmar, eliminando la voz en off, reconociendo la agencia local, está dando lugar a una nueva forma de crear relatos, una forma en la que la horizontalidad substituye a la verticalidad.

El caso guna ilustra cómo dentro de los procesos de lucha y resistencia indígena, el acceso y control de las 
nuevas tecnologías de información y comunicación ha ido cobrando importancia e incluso ha creado nuevos espacios en los que negociar las identidades (Salazar 2002: 64). También muestra hasta qué punto estas tecnologías se adaptan a las necesidades políticas y a las estructuras sociales existentes (Michaels y Kelly 1984; Franco 2016).

\section{JUAN SANT, EL RAP TOTONACO}

Juan Sant, joven indígena totonaco originario de Pantepec (Sierra norte de Puebla), llegó a México D.F. con 15 años y en su proceso de adaptación a las calles y a su nueva realidad con pandillas de cholos descubrió el hip hop y el rap chicano como vías de expresión y creación. Sus letras en totonaco y español hablan de discriminación, de precariedad, pero también de orgullo indígena -su primer disco se llama «El ego de un indio» (2014) $)^{11}$ - y adaptación, y van dirigidas sobre todo a migrantes de origen indígena, a urbanitas y jóvenes indígenas en sus territorios, emergiendo una difusa comunidad política y estética de orden translocal que comparte el hip hop como lengua común con el mundo global, y un grado rudimentario o avanzado de lengua indígena, como seña de identificación.

Su figura ha sido objeto de tesis de licenciatura en Comunicación y Cultura (Hernández 2014) o en Literatura Hispánica sobre Slam Poetry (Garduño 2019: 66-67), de un artículo en una revista de comunicación brasileña (Nascimento 2019), o un capítulo etnográfico sobre Cuautepec (Pineda 2019). En prácticamente todos los textos mencionados aparece siendo entrevistado, inclusive en youtube ${ }^{12}$.

Durante la Colonia española, las autoridades indígenas totonacas fueron los intermediarios entre sus comunidades y la administración colonial (Chenaut 2010: 53), continuando hasta tiempos presentes como mediadores intracomunitarios. Junto a la aparición de los primeros profesionales totonacos, la implantación de la Universidad Veracruzana Intercultural (UVI) en Espinal (Veracruz), ha generado promociones de egresados como gestores culturales que desarrollan su actividad como mediadores con el sistema mexicano de justicia, entre sus médicos tradicionales y el sistema de salud nacional, o entre cultivadores de maíz e

11 <https://www.youtube.com/watch?v=spK9rwZlqy8>.

12 <https://www.youtube.com/watch?v=FsXY5wYypgQ>. ingenieros agrónomos (Dietz 2012: 188). Según García Martínez (2012: 86) el surgimiento de una «juventud indígena» entre los totonacos supondría en ellos la asunción de tareas de mediación en su reformulación identitaria - tomar lo de ellos y ser yo mismo».

En sus videos ${ }^{13}$ (producidos por Mente Negra) se detecta un gusto por la descontextualización. Inserta figuras rituales enmascaradas en escenas y escenarios de la vida urbana donde transcurren los vídeos, como Cuautepec, barrio del Defe. En "Quit (Yo soy)» ${ }^{14}$, un personaje disfrazado de santiaguero, y en "Somos» ${ }^{15}$, un diablo rojo de las danzas de huehuenches y diablos.

La parte inicial del vídeo "Quit (Yo soy)» es una danza de los santiagueros -una danza correspondiente a la categoría de las danzas de Conquista-, originaria de Pantepec (Puebla), donde el danzante aparece con su máscara y atuendo tradicional, escudo y machete, en la sierra, y calzando unas Nike. Los comentarios al vídeo, son realizados en español e inglés pero también en totonaco, algunos por parte de jóvenes migrantes indígenas en Estados Unidos (New Jersey, Texas), aludiendo a sus raíces e inclusive haciendo referencia a representaciones de tipo panindígena: "Brabo juan sant representas todas nuestras étnicas» (Valentín Cruz). En otro comentario, mezclando totonaco y español, Sheiran Reyes dice: "tonii ku'va! buenísima aportación, no me canso de escucharla [...] kuta'vi tuni-ni, nakunumi no'o». Patti Chumi, mixteca de Oaxaca, hace su comentario en inglés: "This video is beautiful hermano! We are lucky to know our indigenous languages!!!! Soy Mixtec de Oaxaca and I speak Mixteco.I happened to come across your video bro and really enjoyed your music».

Como mediador y artista, Juan Sant evidencia una alta versatilidad en sus performances dependiendo del público al que va dirigido su actuación. En 2019 en la Cumbre Tajín "Festival de la Identidad» ${ }^{16}$ en un vídeo subido a su facebook ${ }^{17}$ de su performance de «Quit (Yo

13 <https://www.youtube.com/playlist?list=PLBUoDZuE7wConOSKjBBFoHfWQaztxs8nV>.

14 <https://www.youtube.com/watch?v=taUJ1kW9YQ8>.

15 <https://www.youtube.com/watch?v=kXFNyS_ISqc>.

16 Zúñiga (2014:176) nos proporciona una etnografía crítica de la Cumbre Tajín y su "proceso de regeneración cultural" totonaca.

17 <https://www.facebook.com/OSKARR.elias.9/videos/1193184724221568)>. En su página de Facebook (<https://www.facebook.com/juan.sant2>) se presenta como "Juan Sant. Performing artist, Slamer, MC y Escritor en lengua Tutunaku». 
soy)», mantiene su inicial danza de santiaguero. Si en el vídeo original el artista exhibía una ropa y estética hip hopera, con gorra y camiseta con mensaje «I.W.A.» (Indígenas With Attitude) intentando conectar con jóvenes indígenas en ámbitos urbanos, en este contexto de sitio arqueológico aparece luciendo un traje de manta blanco y sombrero de paja como «campesino indígena», esta vez buscando conectar con un público, preferentemente no indígena, de masas.

Más que una herramienta de afirmación y resistencia étnica, el rock indígena (García Martínez 2014: 56), y en el caso de Juan Sant el rap originario producidos desde Totonacapan, implicarían una reinvención de subjetividades a partir de la experiencia migratoria, centrada en un sistema sociolingüístico móvil, que refleja diferentes escalas de diversidad (De León 2018: 43).

Estas músicas compartidas en las redes sociales, conforman etnopaisajes virtuales (Appadurai 1996: 33; Mendoza Denton 2016) policéntricos, con usuarios y comentarios realizados en espacios translocales y transnacionales, pero donde la música es el foco centrípeto sobre el que se produce el intercambio.

\section{CONCLUSIONES}

Los tres casos anteriores ilustran la emergencia de una juventud indígena que asume un rol protagónico en la era digital. Iván Jaripio, los hermanos Wagua y Juan Sant se convierten en mediadores que crean relatos comprensibles tanto para las audiencias indígenas de las comunidades locales, eminentemente rurales, como para los migrantes o indígenas urbanos, así como para audiencias globales no indígenas. En este sentido estos artistas, a la manera de nuevos médiums, practican una mediación que implica la presencia de aquello que está ausente (Meyer 2011). De esta manera generan unas representaciones novedosas de lo indígena en las que, por otra parte, las voces femeninas son todavía incipientes.

Los casos también ponen en evidencia que donde hay derechos territoriales consolidados la mediación es más fuerte: implica más control sobre el acceso a las comunidades, las filmaciones y la representación, tal y como hacen tanto el Congreso General Guna como Wagua Films.

¿Cómo entender el rol de los cineastas y raperos indígenas? No son una representación del grupo como sucede con los líderes tradicionales, es decir, no tienen una relación metonímica con su gente, cuando su imagen se daña no lo hace la de su grupo. Tampoco encajan en la distinción que hizo Gershon (2006) a partir del estudio de los mediadores samoanos de San Francisco entre el mediador traductor y el mediador representante. Los cineastas y raperos no son ni traductores ni representantes de su pueblo, no pueden traducir su conocimiento tradicional porque los sabios y los jefes locales no creen pertinente compartir estos conocimientos con los blancos, pero sí pueden construir una realidad comprensible desde dentro y para fuera y mostrar la lucha de su pueblo por el territorio y la cultura explicando al mundo, desde el middle ground audiovisual, que van a seguir resistiendo.

\section{BIBLIOGRAFÍA CITADA}

Appadurai, Arjun. 1996. Modernity at Large: Cultural Dimensions of Globalization. Minneapolis: University of Minnesota Press.

Chenaut, Victoria. 2010. «Los totonacas de Veracruz: Población, cultura y sociedad». En Enrique Florescano y Juan Ortiz Escamilla (eds.), Atlas del Patrimonio Natural, Histórico y Cultural de Veracruz: 46-66. Xalapa: Gobierno del Estado de Veracruz/Universidad Veracruzana.

Coleman, E. Gabriella. 2010. «Ethnographic Approaches to Digital Media». Annual Review of Anthropology 39: 487-505. doi: <https://doi.org/10.1146/annurev. anthro.012809.104945>.

Colin, France-Lise. 2010. Nosotros no Solamente Podemos Vivir de Cultura: Identity, Nature and Power in the Comarca Emberá of Eastern Panama. Tesis doctoral. Carleton University.

Conklin, Beth A. y Laura R. Graham. 1995. "The Shifting Middle Ground: Amazonian Indians and Eco-Politics». American Anthropologist 97(4): 695-710. doi: <https://doi. org/10.1525/aa.1995.97.4.02a00120>.

De León-Pasquel, Lourdes. 2018: «Entre el mensaje romántico $y$ el etnorock en YouTube: repertorios identitarios en los paisajes virtuales de jóvenes mayas tsotsiles». Revista LiminaR. Estudios Sociales y Humanísticos 16 (1): 40-55. doi: <https://doi.org/10.29043/liminar.v16i1.563>.

Dietz, Gunther. 2012. «Diversity Regimes Beyond Multiculturalism? A Reflexive Ethnography of Intercultural Higher Education in Veracruz, Mexico». Latin American and Caribbean Ethnic Studies 7 (2): 173-200. doi: <https://doi.or $\mathrm{g} / 10.1080 / 17442222.2012 .686334>$.

Evans, Michelle M. 2012. Be:Longing - Enacting Indigenous Arts Leadership. Tesis doctoral. The University of Melbourne.

Evans, Michelle M. y Amanda Sinclair. 2016. «Navigating the Territories of Indigenous Leadership: Exploring the 
Experiences and Practices of Australian Indigenous Arts Leaders». Leadership 12(4): 470-490. doi: <https://doi. org/10.1177/1742715015574318>.

Franco Coelho, Rafael.2016. La apropiación cultural, social y política de los medios de comunicación en comunidades indígenas: el proyecto aldea digital en el pueblo xavante (Brasil central). Tesis doctoral. Universitat Autònoma de Barcelona.

García Martínez, Ariel. 2012. «Juventud indígena en el Totonacapan veracruzano». Revista LiminaR. Estudios sociales y humanísticos 10 (1): 75-88. doi: <https://doi. org/10.29043/liminar.v10i1.38>

García Martínez, Ariel. 2014. «Rock y juventudes indígenas en el Totonacapan». En Martín de la Cruz López Moya, Efraín Ascencio Cedillo y Juan Pablo Zebadua Carbonell (coords.), Etnorock: Los rostros de una música global en el sur de México: 43-57. México: Cesmeca/Juan Pablo Editores.

Garduño, María Y. 2019. Slam Poetry: una comparativa entre México y Estados Unidos. Tesis de Licenciatura. Benemérita Universidad Autónoma de Puebla.

Gershon, Ilana.2006. «When Culture is not a System. whySamoan Cultural Brokers can not do their Job». Ethnos 71 (4): 533558. doi: <https://doi.org/10.1080/00141840601050700>.

Ginsburg, Faye. 2002. «Mediating Culture: Indigenous Media, Ethnographic Film and the Production of Identity». En Kelly Askew y Richard R. Wilk (eds.), The Anthropology of Media: A Reader: 210-235. Oxford: Blackwell Publishers.

Gómez Ruiz, Sebastián y Gabriel Izard Martínez. 2020. «Indigeneidad performada. Apuntes etnográficos de dos festivales de cine indígena en Colombia y Panamá». Revista Española de Antropología Americana 50: 265-276. doi: <https://doi.org/10.5209/reaa.70379>.

Herlihy, Peter H. 1986. A Cultural Geography of the Embera and Wounan (Choco) Indians of Darien, Panama, with Emphasis on Recent Village Formation and Economic Diversification. Tesis doctoral. Louisiana State University.

Hernández Mejía, Nicolás. 2014. Resignificación del Hip Hop en los jóvenes indígenas migrantes en la Ciudad de México. La experiencia de Juan Sant. Tesis de Licenciatura. Universidad Católica de Murcia México.

Kenny, Carolyn y Tina N. Fraser. 2013. Living Indigenous Leadership: Native Narratives on Building Strong Communities. Vancouver: University of British Columbia Press.

López García, Julián. 2012. «Teléfonos celulares en la era de los mayas: representaciones y usos entre los ch'ort'i de Guatemala». En Pedro Pitarch y Gemma Orobitg (eds.), Modernidades indígenas: 89-114. Madrid/ Frankfurt: Iberoamericana/Vervuert. doi: <https://doi. org/10.31819/9783954870035>.

Martínez Mauri, Mònica. 2011. La autonomía indígena en Panamá. La experiencia del pueblo kuna (siglos XVI-XX). Quito: Editorial Abya Yala.
Mendoza Denton, Norma. 2016. «Norteño and Sureño Gangs, Hip Hop, and Ethnicity in YouTube. Localism in California through Spanish Accent Variation». En H. Samy Alim, John R. Rickford y Arnetha F. Ball (coords.), Raciolinguistics: How Language Shapes our Ideas about Race: 135-152. Oxford: Oxford University Press. doi: <https://doi.org/10.1111/jola.12286>.

Meyer, Birgit. 2011. «Mediation and Immediacy: Sensational Forms, Semiotic Ideologies and the Question of the Medium». Social Anthropology 19 (1): 23-39. doi: <https:// doi.org/10.1111/j.1469-8676.2010.00137.x>.

Michaels, Eric y Francis J. Kelly. 1984. «The Social Organization of an Aboriginal Video Workplace». Australian Aboriginal Studies 1: 26-34.

Nascimento, Roberta Marques. 2019. «Slam, eslameros e outras cumbias: uma experiência no Circuito Nacional de Poesia Falada da cidade do México». Revista Nexi 5 [s.p.]. Disponible en: <https://revistas.pucsp.br/index.php/nexi/ article/view/42316>. Fecha de acceso: 10 feb.2021.

Orobitg, Gemma. (2020a). «Introducción al dossier: Etnografía de los medios de comunicación indígenas y afroamericanos: propuestas metodológicas». Revista Española de Antropología Americana 50: 211-214. doi: <https://doi. org/10.5209/reaa.71751>.

Orobitg, Gemma (ed.). 2020b. Medios indígenas. Teorías y experiencias de la comunicación indígena en América Latina. Madrid/Frankfurt: Iberoamericana/Vervuert.

Pineda, Ismael. 2019. «Mundos distantes: diversidad indígena en Cuautepec». En Iván Gomezcésar Hernández y Cuauhtémoc Ochoa Tinoco (coords.), Cuautepec: actores sociales, cultura y territorio: 363-394. México: Universidad Autónoma de la Ciudad de México.

Salazar, Juan Francisco. 2002. «Activismo indígena en América Latina: estrategias para una construcción cultural de las tecnologías de información y comunicación». Journal of Iberian and Latin American Studies 8 (2): 61-79. doi: <https://doi.org/10.1080/13260219.2002.10431783>.

Salazar, Juan Francisco y Amalia Córdova. 2008. «Imperfect Media and the Poetics of Indigenous Video in Latin America». En Pamela Wilson y Michelle Stewart (eds.), Global Indigenous Media: Cultures, Poetics, and Politics: 3957. Durham y Londres: Duke University Press. doi: <https:// doi.org/10.1215 / 9780822388692-003>.

Turner, Terence. 1992. «Defiant Images: The Kayapo Appropriation of Video». Anthropology Today 8 (6): 5-16. doi: <https://doi.org/10.2307/2783265>.

White, Richard. 1991. The Middle Ground: Indians, Empires, and Republics in the Great Lakes Region, 1650-1815. Nueva York: Cambridge University Press.

Zúñiga, Fernando. 2014. «Nuevos usos del patrimonio arqueológico de el tajín, a través de los procesos de turistificación, mercantilización y espectacularización». Anales de Antropología 48 (2): 151-182. doi: <https://doi. org/10.1016/S0185-1225(14)70247-4>. 